\title{
Digital holographic three-dimensional imaging spectrometry based on rotated-hyperbolic type volume interferogram
}

\author{
Masaki Obara* and Kyu Yoshimori \\ Department of Electrical Engineering and Computer Science, Graduate School of Engineering, \\ Iwate University, Morioka 020-8551, Japan
}

\begin{abstract}
This paper presents a new method of digital holographic three-dimensional imaging spectrometry. The method is based on the measurement of new type of volume interferogram, called Rotated-Hyperbolic-type volume interferogram, obtained with the existing interferometer. We report the first demonstration of the method in which a monochromatic point source is used as the measured object. The results include 3-D spatial information and spectral information of a monochromatic point source. This experimental result corresponds to the impulse response function defined over a fourdimensional $(x, y, z, k)$ space. As a result, the method is confirmed to have the superior imaging characteristics in the $z$ direction.
\end{abstract}

Keywords: Digital holography, Interferometric imaging, Spectroscopy, Fourier transforms, Imaging spectrometry, fourdimensional impulse response function, two-wavefront folding interferometer, synthetic aperture technique

\section{INTRODUCTION}

We have investigated the method to obtain both three-dimensional (3-D) spatial information and spectral information of a usual polychromatic object simultaneously without using a special light source and image formation element ${ }^{1}$. It just uses interferometric technique and signal processing. For this purpose, we have suggested a method, called digital holographic three-dimensional imaging spectrometry, which uses a two-wavefront folding interferometer and synthetic aperture technique. Concerning the algorithm, we have proposed two types of those techniques, both of which are based on measurement of five-dimensional (5-D) spatial coherence function. The difference between the two methods lies in the selection rules in which each method uses in the synthetic aperture processing. One rule produces a spherical-type (Stype) volume interferogram ${ }^{2-4}$. The recorded fringe patterns of which directly correspond to the phase distributions of the propagated wavefront of many spectral components from the object. The other rule produces a hyperbolic-type (H-type) volume interferogram. The latter method, i.e., the H-type ${ }^{5}$, gives equivalent results of 3-D image and spectra to the former method using the S-type volume interferogram.

Recently, we have found a new rule for synthetic aperture processing. This rule enables us to create an alternative volume interferogram. The recorded interference patterns in the volume interferogram are not similar to the propagated wavefront from the object.

This paper presents a new method of digital holographic three-dimensional imaging spectrometry. The method is based on the measurement of new type of volume interferogram, called Rotated-Hyperbolic-type (RH-type) volume interferogram, obtained with the existing interferometer, We report the first demonstration of the method in which a monochromatic point source is used as the measured object. The results include 3-D spatial information and spectral information of a monochromatic point source. The experimental result corresponds to the impulse response function defined over a four-dimensional $(x, y, z, k)$ space. Furthermore, we have confirmed that retrieval results with respect to the $z$-direction can be obtained more accurately by this method as compared with two method described above.

\section{PRINCIPLE OF METHOD}

Figure 1 shows the schematic of the two-wavefront folding interferometer that is used in our experimental system. This is an interferometer for measuring the 5-D spatial coherence function. The measured object is set on the $x-y$ stage.

*masaki@ql.cis.iwate-u.ac.jp;

ICPS 2013: International Conference on Photonics Solutions, edited by Prathan Buranasiri, Sarun Sumriddetchkajorn, Proc. of SPIE Vol. 8883, 888319 - @ 2013 SPIE · CCC code: 0277-786X/13/\$18 · doi: 10.1117/12.2022089 


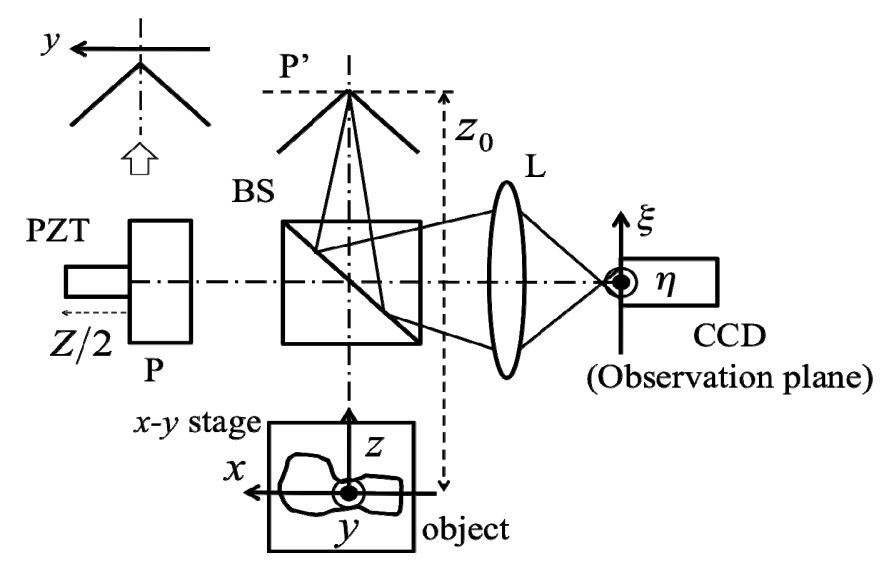

Figure 1. Schematic of experimental system: two-wavefront folding interferometer.

The propagated light wave from the object is split by the beam splitter (BS). The split light waves are reflected by the right-angle-prism P or P'. The two apexes of which are set orthogonally each other. Then, each split light wave is reversed from up and down or right and left by $\mathrm{P}$ or $\mathrm{P}$ '. Those light waves are superposed on BS again. The generated fringe pattern, called the elementally interference pattern, is detected by the two-dimensional (2-D) array detector (CCD). In addition, by the lens (L), the surface of CCD is projected onto the plane containing the apex of the prism P'. This plane is the virtual observation plane. Thus, $z$-distance is measured from this virtual observation plane. For introducing the optical path difference, one of the prisms P may be moved along $z$-axis by the Piezo Translator (PZT). During interferometric measurement, the $x-y$ stage and PZT are moved stepwise. Then, 2-D elementally interference patterns are detected at each position of these stages.

Here, we define a coordinate system $(\xi, \eta)$ of the observation plane, $\hat{x}$ or $\hat{y}$ stand for the $x$ - or $y$-position of the apexes of prism $\mathrm{P}^{\prime}$ or $\mathrm{P}$ each being adjusted by $x-y$ stage, and $Z$ denotes optical path difference introduced by PZT. The 2-D elementally interference patterns obtained by the CCD are arranged as three-dimensionally in $(\hat{x}, \hat{y}, Z)$ space. Then, the whole data set may be regarded as 5-D interferogram.

The spatial coherence function $\Gamma$ contained in the 5-D interferogram is expressed by using the cross-spectral density $W_{k}$,

$$
\Gamma\left(\boldsymbol{r}^{\prime}, \boldsymbol{r}\right)=c \int_{0}^{\infty} W_{\omega}\left(\boldsymbol{r}^{\prime}, \boldsymbol{r}^{\prime \prime}\right) \exp (i k Z) d k
$$

Where, $\boldsymbol{r}=\left(\xi+\hat{x},-\eta+\hat{y}, z_{0}+Z\right), \quad \boldsymbol{r}^{\prime}=\left(-\xi+\hat{x}, \eta+\hat{y}, z_{0}\right)$ and $\boldsymbol{r}^{\prime \prime}=\left(\xi+\hat{x},-\eta+\hat{y}, z_{0}\right)$. Here, $z_{0}$ is the optical length between the virtual observation plane and the origin of the Cartesian coordinate system fixed on the $x-y$ stage, $\omega=c k$ is the angular frequency, $c$ is the speed of light in free space, and $k$ is the wave-number. To compute the reduced volume (3-D) interferogram, we apply the synthetic aperture technique to the 5-D interferogram. We will find this interferogram is suitable for retrieving the source information under observation by the selection rule and the rearrangement. For generating the volume interferogram, we choose one pixel from each elementally interference pattern according to the selection rule and rearrange the selected pixel. By modifying the selection rule, we may create the various patterns of volume interferogram. The presented method, we use the new selection rule. The example of the computed RH-type volume interferogram is shown in Fig. 2.

By taking the Fourier transform with respect to optical path difference $Z$, we obtain the cross-spectral density at each spectral component from the volume interferogram. Since the cross-spectral density is proportional to the complex hologram of monochromatic component, it is possible to retrieve a set of spectral components of 3-D images of the spatially incoherent, polychromatic light source under measurement, from these cross-spectral densities by applying the appropriate retrieval processing. 


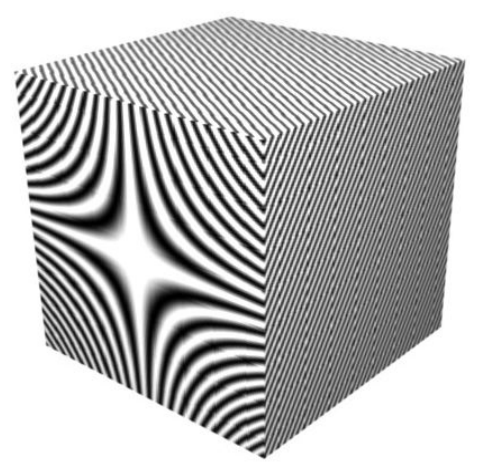

Figure 2. The example of the RH-type volume interferogram computed by the synthetic aperture technique.

\section{EXPERIMENT}

We show the experimental results for retrieving the spectral component of 3-D image of the light source. The measured object is a monochromatic point source, whose wavelength is $543.5 \mathrm{~nm}$. Guiding the He-Ne laser light through a singlemode optical fiber to make this point source. The distance $z$ between the object and virtual observation plane is $93 \mathrm{~mm}$. The $x-y$ stage of steps and PZT are moved stepwise at constant intervals. The numbers of steps and intervals are 32 and $12.9 \mu \mathrm{m}$ for the $x-y$ stage and 64 and $80 \mathrm{~nm}$ for PZT, respectively. As a result, the measured 5-D interferogram is composed of $32 \times 32 \times 64$ elemental interference patterns.

Figures 3(a) and 3(b) show the phase distribution and absolute value of the cross-spectral density at $543.5 \mathrm{~nm}$. From Fig. 3(a), the rotating hyperbolic can be seen as characteristic shape. Therefore, we call it Rotated-Hyperbolic-type. Figure 4 shows the retrieved cross section of the in-focus spectral image over the $x-y$ plane.
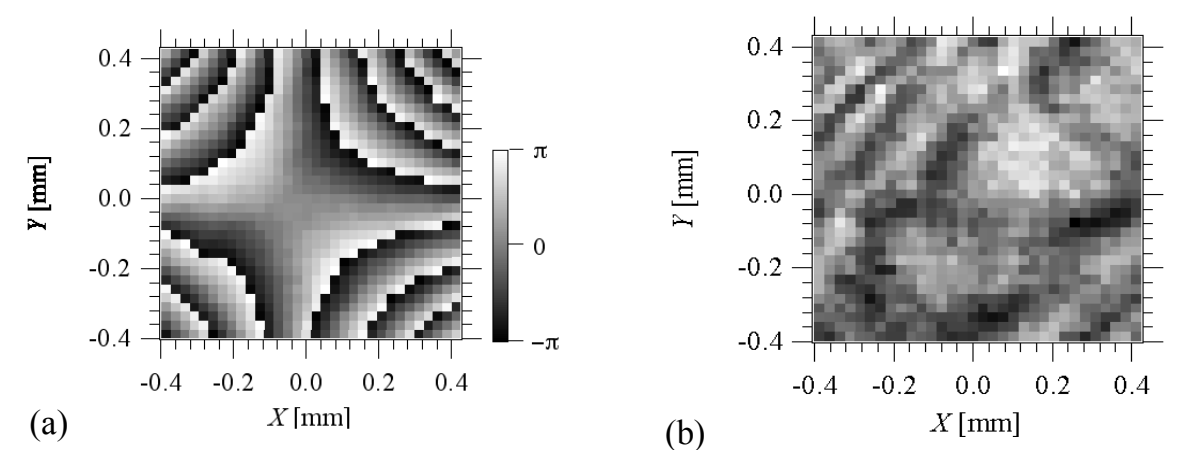

Figure 3. Phase distribution and absolute value of the cross-spectral density at wavelength $=543.5 \mathrm{~nm}$.

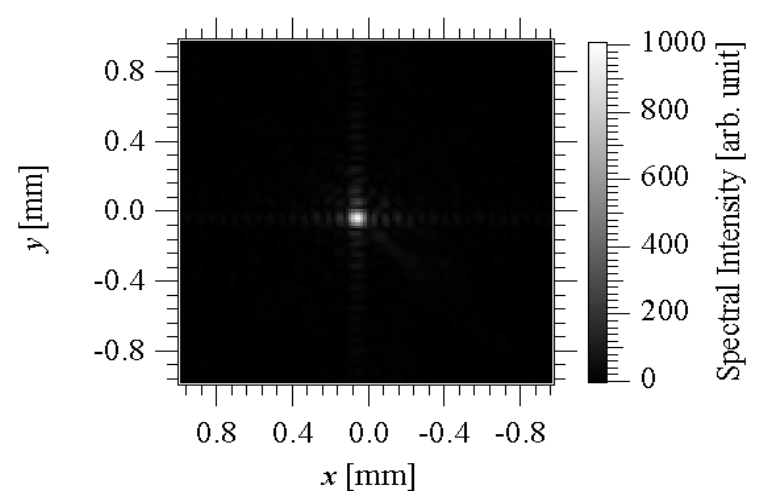

Figure 4. In-focus spectral image of monochromatic point source over $x-y$ plane at $z=93 \mathrm{~mm}$. 
Figure 5(a) and 5(b) shows the intensity profiles along the $x$ - and $y$-axes in Fig. 4. We find that both peaks appear close to the origin. In Figure 6 shows a comparison of the intensity profile along the $z$-axis obtained by the present method and the conventional method. From these experimental results, we demonstrated that 3-D information and spectral information of monochromatic point source can be successfully acquired simultaneously.

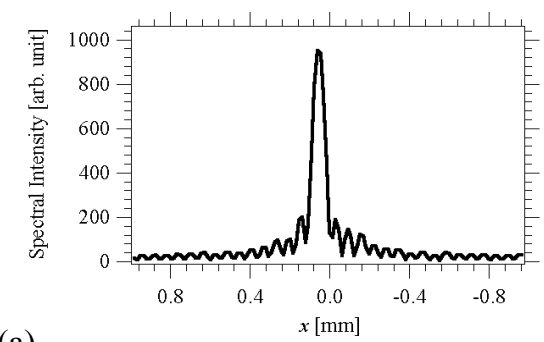

(a)

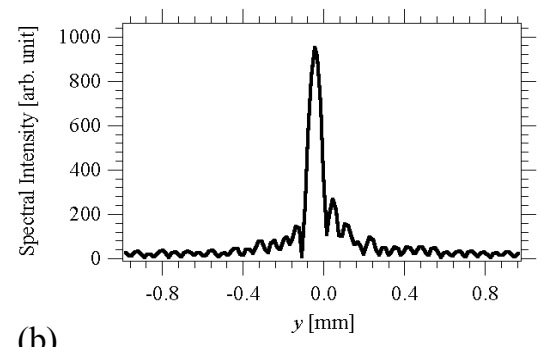

(b)

Figure 5. Intensity profiles of in-focus image (shown in Fig. 4) along (a) $x$ - and (b) $y$-axes.

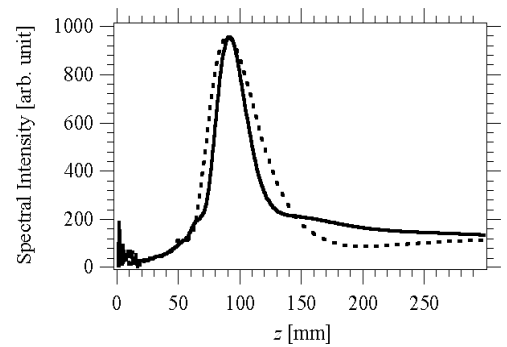

Figure 6. Intensity profile of in-focus image along $z$-axis. The solid line is the result of the present method; the broken line is the result of the conventional method using S-type volume interferogram.

\section{CONCLUSION}

This paper presented a new method of digital holographic three-dimensional imaging spectrometry. The method is based on the measurement of the Rotated-Hyperbolic-type (RH-type) volume interferogram obtained with the existing interferometer. We have showed the experimental result using the monochromatic point source and succeeded in obtaining the 3-D spatial information and spectral information of measured object. As a result, the present method compared to the above two methods that have been reported so far, was confirmed to have the superior imaging characteristics in the $z$-direction.

\section{ACKNOWLEDGMENTS}

This work is supported in part by the Research Foundation for Opto-Science and Technology, and Grants-in-Aid for Scientific Research 25390087 from the Japan Society for the Promotion of Science.

\section{REFERENCES}

[1] Yoshimori K., "Passive digital multispectral holography based on synthesis of coherence function," Proc. SPIE 6252, 625221-1625221-3 (2006).

[2] Sasamoto M. and Yoshimori K., "First Experimental Report on Fully Passive Interferometric ThreeDimensional Imaging Spectrometry," Jpn. J. Appl. Phys. 48, 09LB03-1-09LB03-4 (2009).

[3] Sasamoto M. and Yoshimori K., "Three-Dimensional Imaging Spectrometry by Fully Passive Interferometry," Opt. Rev. 19, 29-33 (2012).

[4] Teeranutranont S. and Yoshimori K., "Digital holographic three-dimensional imaging spectrometry," Appl. Opt. 52, A388-A396 (2012).

[5] Hashimoto T. Hirai A. and Yoshimori K., "Fully interferometric three-dimensional imaging spectrometry using hyperbolic-type volume interferogram," Appl. Opt. 52, 1497-1504 (2013). 